\title{
The $\Sigma^{0} \Lambda$ Transition Magnetic Moment in Light Cone QCD Sum Rules
}

\author{
T. M. Aliev *, A. Özpineci ${ }^{\dagger}$, M. Savc1 ${ }^{\ddagger}$ \\ Physics Department, Middle East Technical University \\ 06531 Ankara, Turkey
}

\begin{abstract}
Using the general form of the $\Sigma^{0}$ and $\Lambda$ currents, the $\Sigma^{0} \Lambda$ transition magnetic moment is calculated in framework of the light cone QCD sum rules. A comparison of our result on this quantity with the existing theoretical results and experimental data is presented.
\end{abstract}

PACS number(s): 11.55.Hx, 13.40.Em, 14.20.Jn

*e-mail: taliev@metu.edu.tr

$\dagger$ e-mail: altugoz@metu.edu.tr

‡e-mail: savci@metu.edu.tr 


\section{Introduction}

Determination of the various fundamental parameters of hadrons from experiments requires information about physics at large distance. Unfortunately such information can not be achieved from the first principles of a fundamental theory of strong interactions because at large distance perturbation theory is invalid. For this reason, to determine properties of hadrons a reliable nonperturbative approach is needed. Among all nonperturbative approaches, QCD sum rules [1] method is an especially powerful one in studying the properties low-lying hadrons. In this method, deep connection between hadron parameters and QCD vacuum structure is established via a few condensates. This method is adopted and extended in many works (see for example $[2,3,4]$ and references therein). One of the important characteristics of hadrons is their magnetic moment. The nucleon magnetic moment and the $\Sigma^{0} \Lambda$ transition magnetic moment were calculated using the external field technique in framework of the QCD sum rules method, in [5, 6] and [7], respectively.

The aim of this letter is to calculate the $\Sigma^{0} \Lambda$ transition magnetic moment in framework of an alternative approach to the traditional sum rules method, i.e., light cone QCD sum rules (LCQSR) (more about LCQSR method and its applications can be found in [8, 9] and references therein). Note that magnetic moment of the nucleons and decuplet baryons were studied in the LCQSR approach in [10] and [11, 12] respectively. The paper is organized as follows: In Sect. 2, the LCQSR for the $\Sigma^{0} \Lambda$ magnetic moment is derived. Sect. 3 is devoted to our numerical analysis and conclusion.

\section{Sum Rules for the $\Sigma^{0} \Lambda$ Transition Magnetic Moment}

For the determination of the $\Sigma^{0} \Lambda$ transition magnetic moment in LCQSR, we consider the following two point correlation function:

$$
\Pi_{\Sigma^{0} \Lambda}=i \int d^{4} x e^{i p x}\left\langle 0\left|T\left\{\eta_{\Sigma^{0}}(x) \bar{\eta}_{\Lambda}(0)\right\}\right| 0\right\rangle_{\gamma}
$$

where $T$ is the time ordering operator, $\gamma$ means external electromagnetic field and $\eta_{\Sigma^{0}}$, $\eta_{\Lambda}$ are the interpolating currents with $\Sigma^{0}$ and $\Lambda$ quantum numbers,respectively. It is well known that there is a continuum of choices for the baryon interpolating current. The general form of the $\Sigma^{0}$ and $\Lambda$ currents can be written as $[4,13]$ :

$$
\begin{aligned}
& \eta_{\Sigma^{0}}=2\left(\eta_{\Sigma_{1}}+t^{\prime} \eta_{\Sigma_{2}}\right) \\
& \eta_{\Lambda^{0}}=2\left(\eta_{\Lambda_{1}}+t \eta_{\Lambda_{2}}\right)
\end{aligned}
$$

where $t$ and $t^{\prime}$ are arbitrary parameters and

$$
\begin{aligned}
\eta_{\Sigma_{1}} & =\frac{1}{\sqrt{2}} \epsilon_{a b c}\left[\left(u_{a}^{T} C s_{b}\right) \gamma_{5} d_{c}+\left(d_{a}^{T} C s_{b}\right) \gamma_{5} u_{c}\right], \\
\eta_{\Sigma_{2}} & =\frac{1}{\sqrt{2}} \epsilon_{a b c}\left[\left(u_{a}^{T} C \gamma_{5} s_{b}\right) d_{c}+\left(d_{a}^{T} C \gamma_{5} s_{b}\right) u_{c}\right], \\
\eta_{\Lambda_{1}} & =\frac{1}{\sqrt{6}} \epsilon_{a b c}\left[2\left(u_{a}^{T} C d_{b}\right) \gamma_{5} s_{c}+\left(u_{a}^{T} C s_{b}\right) \gamma_{5} d_{c}-\left(d_{a}^{T} C s_{b}\right) \gamma_{5} u_{c}\right],
\end{aligned}
$$




$$
\eta_{\Lambda_{2}}=\frac{1}{\sqrt{6}} \epsilon_{a b c}\left[2\left(u_{a}^{T} C \gamma_{5} d_{b}\right) s_{c}+\left(u_{a}^{T} C \gamma_{5} s_{b}\right) d_{c}-\left(d_{a}^{T} C \gamma_{5} s_{b}\right) u_{c}\right]
$$

where $a, b$, and $c$ are color indices. Ioffe current corresponds to the choice $t=t^{\prime}=-1$.

Firstly, let us discuss the hadronic representation for the correlator. This can be done by inserting a complete set of one hadron states into the correlator:

$$
\Pi_{\Sigma^{0} \Lambda}=\sum \frac{\left\langle 0\left|\eta_{\Sigma^{0}}\right| B_{1}\left(p_{1}\right)\right\rangle}{p_{1}^{2}-M_{1}^{2}}\left\langle B_{1}\left(p_{1}\right) \mid B_{2}\left(p_{2}\right)\right\rangle_{\gamma} \frac{\left\langle B_{2}\left(p_{2}\right)\left|\bar{\eta}_{\Lambda}\right| 0\right\rangle}{p_{2}^{2}-M_{1}^{2}}
$$

where $p_{2}=p_{1}+q, q$ is the photon momentum, $B_{i}$ form a complete set of baryons having the same quantum numbers as $B$ with masses $M_{i}$.

The interpolating current couples to the baryon states with the overlap amplitudes $\lambda$ defined by:

$$
\begin{array}{r}
\left\langle 0\left|\eta_{\Sigma^{0}}\right| \Sigma^{0}\right\rangle=\lambda_{\Sigma^{0}} u_{\Sigma^{0}}(p) \\
\left\langle 0\left|\eta_{\Lambda}\right| \Lambda\right\rangle=\lambda_{\Lambda} u_{\Lambda}(p)
\end{array}
$$

It follows from Eq. (7) that in order to write down the phenomenological part of the correlator, an expression for the matrix element $\left\langle\Sigma^{0}\left(p_{1}\right) \mid \Lambda\left(p_{2}\right)\right\rangle_{\gamma}$ is needed. This matrix element can be written as:

$$
\begin{aligned}
\left\langle\Sigma^{0}\left(p_{1}\right) \mid \Lambda\left(p_{2}\right)\right\rangle_{\gamma} & =\bar{u}\left(p_{1}\right)\left[f_{1} \gamma_{\mu}+i \frac{\sigma_{\mu \alpha} q^{\alpha}}{m_{\Sigma^{0}}+m_{\Lambda}} f_{2}\right] u\left(p_{2}\right) \varepsilon^{\mu} \\
& =\bar{u}\left(p_{1}\right)\left[\left(f_{1}+f_{2}\right) \gamma_{\mu}+\frac{\left(p_{1}+p_{2}\right)_{\mu}}{m_{\Sigma^{0}}+m_{\Lambda}} f_{2}\right] u\left(p_{2}\right) \varepsilon^{\mu}
\end{aligned}
$$

where the form factors $f_{i}$ are in general functions of $q^{2}=\left(p_{2}-p_{1}\right)^{2}$ and $\varepsilon^{\mu}$ is the polarization four vector of the photon. In our case, in order to evaluate the transition magnetic moment, only the value of the form factors at $q^{2}=0$ are needed.

Using Eqs. (7), (8), and (9), for the phenomenological part of the LCQSR we get:

$$
\Pi_{\Sigma^{0} \Lambda}=-\lambda_{\Sigma^{0}} \lambda_{\Lambda} \varepsilon^{\mu} \frac{\not p_{1}+m_{\Sigma^{0}}}{p_{1}^{2}-m_{\Sigma^{0}}^{2}}\left[\left(f_{1}+f_{2}\right) \gamma_{\mu}+\frac{\left(p_{1}+p_{2}\right)_{\mu}}{m_{\Lambda}+m_{\Sigma^{0}}} f_{2}\right] \frac{\not p_{2}+m_{\Lambda}}{p_{2}^{2}-m_{\Lambda}^{2}} .
$$

Obviously, it follows from this expression that the correlator function contains a number of different structures. Among all possible structures, we choose the one $\sim \not p_{1} \not k \not q$ that contains the transition magnetic form factor $f_{1}+f_{2}$, which when evaluated at $q^{2}=0$ gives the transition magnetic moment in units of $e \hbar /\left(m_{\Lambda}+m_{\Sigma^{0}}\right)$. Isolating the structure $\sim \not p_{1} \not \not p_{2}$ from the phenomenological part of the correlator, which describes the $\Sigma^{0} \Lambda$ transition form factor, we get

$$
\Pi_{\Sigma^{0} \Lambda}=-\lambda_{\Sigma^{0}} \lambda_{\Lambda} \frac{1}{p_{1}^{2}-M_{\Sigma^{0}}^{2}} \mu_{\Sigma^{0} \Lambda} \frac{1}{p_{2}^{2}-m_{\Lambda}^{2}}
$$

where $\mu_{\Sigma^{0} \Lambda}=\left.\left(f_{1}+f_{2}\right)\right|_{q^{2}=0}$.

Calculation of the correlator function $\Pi_{\Sigma^{0} \Lambda}$ from the QCD side leads to the following result: 


$$
\begin{aligned}
& \Pi_{\Sigma^{0} \Lambda}\left(p_{1}^{2}, p_{2}^{2}\right)=-\frac{2}{\sqrt{3}} \epsilon^{a b c} \epsilon^{d e f} \int d^{4} x e^{i p x} \\
& \langle\gamma|\left\{2 \gamma_{5} S_{d}^{c f} S_{u}^{\prime a d} S_{s}^{b e} \gamma_{5}+2 t \gamma_{5} S_{d}^{c f} \gamma_{5} S_{u}^{\prime a d} S_{s}^{b e}\right. \\
& +2 t^{\prime} S_{d}^{c f} S_{u}^{\prime a d} \gamma_{5} S_{s}^{b e} \gamma_{5}+2 t t^{\prime} S_{d}^{c f} \gamma_{5} S_{u}^{\prime a d} \gamma_{5} S_{s}^{b e} \\
& +\gamma_{5} S_{d}^{c f} \gamma_{5} \operatorname{Tr} S_{u}^{a d} S_{s}^{\prime} b e+t \gamma_{5} S_{d}^{c f} \operatorname{Tr} S_{u}^{a d} \gamma_{5} S_{s}^{\prime b e} \\
& +t^{\prime} S_{d}^{c f} \gamma_{5} \operatorname{Tr} S_{u}^{a d} S_{s}^{\prime b e} \gamma_{5}+t t^{\prime} S_{d}^{c f} \operatorname{Tr} S_{u}^{a d} \gamma_{5} S_{s}^{\prime b e} \gamma_{5} \\
& -\gamma_{5} S_{d}^{c f} S_{s}^{\prime b e} S_{u}^{a d} \gamma_{5}-t \gamma_{5} S_{d}^{c f} \gamma_{5} S_{s}^{\prime b e} S_{u}^{a d} \\
& -t^{\prime} S_{d}^{c f} S_{s}^{\prime b e} \gamma_{5} S_{u}^{a d} \gamma_{5}-t t^{\prime} S_{d}^{c f} \gamma_{5} S_{s}^{\prime b e} \gamma_{5} S_{u}^{a d} \\
& -2 \gamma_{5} S_{u}^{a d} S_{d}^{\prime c f} S_{s}^{b e} \gamma_{5}-2 t \gamma_{5} S_{u}^{a d} \gamma_{5} S_{d}^{\prime c f} S_{s}^{b e} \\
& -2 t^{\prime} S_{u}^{a d} S_{d}^{\prime c f} \gamma_{5} S_{s}^{b e} \gamma_{5}-2 t t^{\prime} S_{u}^{a d} \gamma_{5} S_{d}^{\prime c f} \gamma_{5} S_{s}^{b e} \\
& +\gamma_{5} S_{u}^{a d} S_{s}^{\prime b e} S_{d}^{c f} \gamma_{5}+t \gamma_{5} S_{u}^{a d} \gamma_{5} S_{s}^{\prime b e} S_{d}^{c f} \\
& +t^{\prime} S_{u}^{a d} S_{s}^{\prime b e} \gamma_{5} S_{d}^{c f} \gamma_{5}+t t^{\prime} S_{u}^{a d} \gamma_{5} S_{s}^{\prime b e} \gamma_{5} S_{d}^{c f} \\
& -\gamma_{5} S_{u}^{a d} \gamma_{5} \operatorname{Tr} S_{d}^{\prime c f} S_{s}^{b e}-t \gamma_{5} S_{u}^{a d} \operatorname{Tr} \gamma_{5} S_{d}^{\prime c f} S_{s}^{b e} \\
& \left.-t^{\prime} S_{u}^{a d} \gamma_{5} \operatorname{Tr} S_{d}^{\prime c f} \gamma_{5} S_{s}^{b e}-t t^{\prime} S_{u}^{a d} \operatorname{Tr} \gamma_{5} S_{d}^{\prime c f} \gamma_{5} S_{s}^{b e}\right\}|0\rangle \text {, }
\end{aligned}
$$

where $S^{\prime}=C S^{T} C$. Here $C$ is the charge conjugation operator and $T$ denotes transpose of the operator.

In order to obtain the perturbative contribution (i.e., photon is radiated from the freely propagating quarks) it is enough to make the following substitution in one of the propagators in Eq. (12)

$$
S_{q_{\alpha \beta}}^{a b} \rightarrow 2\left(\int d y F^{\mu \nu} y_{\nu} S_{q}^{\text {free }}(x-y) \gamma_{\mu} S_{q}{ }^{\text {free }}(y)\right)_{\alpha \beta}^{a b}
$$

where the Fock-Schwinger gauge $x_{\mu} A^{\mu}(x)=0$ is used and $S_{q}^{\text {free }}$ is the free quark propagator, i.e.

$$
S_{q}^{f r e e}=\frac{i \not x}{2 \pi^{2} x^{4}}
$$

and the remaining two propagators are the full quark propagators (see below).

The expression for nonperturbative contributions can be obtained from Eq. (12) by the following trick: In one of the propagators, we made the replacement

$$
S_{q_{\alpha \beta}}^{a b} \rightarrow-\frac{1}{4} \bar{q}^{a} A_{j} q^{b}\left(A_{j}\right)_{\alpha \beta}
$$

where $A_{j}=\left\{1, \gamma_{5}, \gamma_{\alpha}, i \gamma_{5} \gamma_{\alpha}, \sigma_{\alpha \beta} / \sqrt{2}\right\}$ and sum over $A_{j}$ is implied. For the other two propagators, we substitute the full propagator with both perturbative and nonperturbative contributions.

The complete light cone expansion of the light quark propagator in external field is calculated in [14]. It gets contributions from the $\bar{q} G q, \bar{q} G G q, \bar{q} q \bar{q} q$ nonlocal operators 
(where $G_{\mu \nu}$ is the gluon field strength tensor). In the present work we consider operators with only one gluon field and neglect components with two gluon and four quark fields. Formally, neglect of the $\bar{q} G G q$ and $\bar{q} q \bar{q} q$ terms can be justified on the basis of an expansion in conformal spin [15]. In this approximation full light quark propagator is

$$
\begin{aligned}
S_{q} & =\frac{i \not x}{2 \pi^{2} x^{4}}-\frac{\langle\bar{q} q\rangle}{12}-\frac{m_{q}}{4 \pi^{2} x^{2}}+\frac{i m_{q}\langle\bar{q} q\rangle}{48} \not x-\frac{x^{2}}{192} m_{0}^{2}\langle\bar{q} q\rangle-\frac{i m_{0}^{2} m_{q}}{2^{7} 3^{2}} x^{2} \not x\langle\bar{q} q\rangle \\
& -i g_{s} \int_{0}^{1} d v\left[\frac{\not x}{16 \pi^{2} x^{2}} G^{\mu \nu}(v x) \sigma_{\mu \nu}-v x_{\mu} G^{\mu \nu}(v x) \gamma_{\nu} \frac{i}{4 \pi^{2} x^{2}}\right] .
\end{aligned}
$$

In the local part of the propagator, we neglect operators with dimension $d>5$, since they give negligible contribution.

It follows from Eqs. (12)-(16) that in order to calculate QCD part of the sum rules we need matrix elements of nonlocal operators between photon and vacuum state, $\left\langle\gamma(q)\left|\bar{q} A_{i} q\right| 0\right\rangle$. Up to twist-4, the nonzero matrix elements given in terms of the photon wave function are $[15,16,17]$ :

$$
\begin{aligned}
\left\langle\gamma(q)\left|\bar{q} \gamma_{\alpha} \gamma_{5} q\right| 0\right\rangle & =\frac{f}{4} e_{q} \epsilon_{\alpha \beta \rho \sigma} \varepsilon^{\beta} q^{\rho} x^{\sigma} \int_{0}^{1} d u e^{i u q x} \psi(u) \\
\left\langle\gamma(q)\left|\bar{q} \sigma_{\alpha \beta} q\right| 0\right\rangle & =i e_{q}\langle\bar{q} q\rangle \int_{0}^{1} d u e^{i u q x}\left\{\left(\varepsilon_{\alpha} q_{\beta}-\varepsilon_{\beta} q_{\alpha}\right)\left[\chi \phi(u)+x^{2}\left(g_{1}(u)-g_{2}(u)\right)\right]\right. \\
& \left.+\left[q x\left(\varepsilon_{\alpha} x_{\beta}-\varepsilon_{\beta} x_{\alpha}\right)+\varepsilon x\left(x_{\alpha} q_{\beta}-x_{\beta} q_{\alpha}\right)\right] g_{2}(u)\right\}
\end{aligned}
$$

where $\chi$ is the magnetic susceptibility of the quark condensate, $e_{q}$ is the quark charge, the functions $\phi(u)$ and $\psi(u)$ are the leading twist-2 photon wave functions, while $g_{1}(u)$ and $g_{2}(u)$ are the twist -4 functions. Note that in the calculations, the masses of the $u$ and $d$ quarks are neglected and only the terms linear in the strange quark mass are taken into account. Therefore, under $\mathrm{SU}(2)$ symmetry $u$ and $d$ quark propagators and their condensates are identical, i.e., $\langle\bar{u} u\rangle=\langle\bar{d} d\rangle$ (the difference in the wave functions are due to their charges only).

Substituting the photon wave functions and the expression for the quark propagator into Eq. (12), we can calculate the theoretical part, i.e., OPE part of the correlator (1). The sum rules is obtained by equating the phenomenological and theoretical parts of the correlator. In order to suppress the contributions of the higher states and the continuum, we perform double Borel transformations on the variables $p_{1}^{2}=p^{2}$ and $p_{2}^{2}=(p+q)^{2}$ (for more details see $[11,12,18,19])$, and we get the following result for the transition magnetic moment

$$
\begin{aligned}
& \sqrt{3} \lambda_{\Lambda} \lambda_{\Sigma^{0}} \mu_{\Sigma^{0} \Lambda} e^{-\left(\frac{M_{\Lambda}^{2}}{M_{1}^{2}}+\frac{M_{\Sigma^{0}}^{2}}{M_{2}^{2}}\right)}=\left(e_{d}-e_{u}\right)\left\{-\frac{m_{s}}{16 \pi^{2}}\langle\bar{q} q\rangle\left[t^{\prime}+t\left(-3+2 t^{\prime}\right)\right] M^{4} E_{1}(x) \chi \varphi\left(u_{0}\right)\right. \\
& +\frac{1}{16 \pi^{2}}\left(2+t+t^{\prime}+2 t t^{\prime}\right) M^{4} E_{1}(x) f \psi\left(u_{0}\right)+ \\
& +\frac{m_{s}}{2 \pi^{2}}\langle\bar{q} q\rangle\left[t^{\prime}+t\left(-3+2 t^{\prime}\right)\right]\left[g_{1}\left(u_{0}\right)-g_{2}\left(u_{0}\right)\right] M^{2} E_{0}(x)
\end{aligned}
$$




$$
\begin{aligned}
& +\frac{1}{6}\langle\bar{q} q\rangle\left[\left(-3 t+t^{\prime}+2 t t^{\prime}\right)\langle\bar{s} s\rangle+2\left(-1+t-t^{\prime}+t t^{\prime}\right)\langle\bar{q} q\rangle\right] M^{2} E_{0}(x) \chi \varphi\left(u_{0}\right) \\
& -\frac{2}{3}\langle\bar{q} q\rangle\left[\left(-3 t+t^{\prime}+2 t t^{\prime}\right)\langle\bar{s} s\rangle+2\left(-1+t-t^{\prime}+t t^{\prime}\right)\langle\bar{q} q\rangle\right]\left[g_{1}\left(u_{0}\right)-g_{2}\left(u_{0}\right)\right] \\
& +\frac{m_{s}}{12}\left[\left(2+t+t^{\prime}+2 t t^{\prime}\right)\langle\bar{s} s\rangle+\left(2+t+t^{\prime}-4 t t^{\prime}\right)\langle\bar{q} q\rangle\right] f \psi\left(u_{0}\right) \\
& -\frac{1}{144} m_{0}^{2}\langle\bar{q} q\rangle\left[\left(-4-11 t+5 t^{\prime}+10 t t^{\prime}\right)\langle\bar{s} s\rangle+8\left(-1+t-t^{\prime}+t t^{\prime}\right)\langle\bar{q} q\rangle\right] \chi \varphi\left(u_{0}\right) \\
& -\frac{1}{32 \pi^{4}}\left(2+t+t^{\prime}+2 t t^{\prime}\right) M^{6} E_{2}(x)+\frac{1}{6}\langle\bar{s} s\rangle\langle\bar{q} q\rangle\left(2+t+t^{\prime}-4 t t^{\prime}\right) \\
& -\frac{m_{s}}{8 \pi^{2}} M^{2}\left[\left(2+t+t^{\prime}+2 t t^{\prime}\right)\langle\bar{s} s\rangle+\left(2+t+t^{\prime}-4 t t^{\prime}\right)\langle\bar{q} q\rangle\right] \\
& +\frac{m_{0}^{2} m_{s}}{96 \pi^{2}}\left[2\left(2+t+t^{\prime}+2 t t^{\prime}\right)\langle\bar{s} s\rangle+3\left(2+t+t^{\prime}-4 t t^{\prime}\right)\langle\bar{q} q\rangle\right] \\
& \left.-\frac{3 m_{0}^{2}}{32 \pi^{2}} m_{s}\langle\bar{q} q\rangle\left(1-t t^{\prime}\right)\left(\gamma_{E}-\ln \frac{M^{2}}{\Lambda^{2}}\right)\right\} \\
& -e_{s} \frac{m_{0}^{2}}{96 \pi^{2}} m_{s}\langle\bar{q} q\rangle\left(1-t t^{\prime}\right)\left(\gamma_{E}-\ln \frac{M^{2}}{\Lambda^{2}}\right)
\end{aligned}
$$

where

$$
E_{n}(x)=1-e^{-x} \sum_{0}^{n} \frac{1}{k !} x^{k}
$$

are the functions used to subtract the continuum, $x=s_{0} / M^{2}, s_{0}$ is the continuum threshold and

$$
u_{0}=\frac{M_{2}^{2}}{M_{1}^{2}+M_{2}^{2}}, \quad M^{2}=\frac{M_{1}^{2} M_{2}^{2}}{M_{1}^{2}+M_{2}^{2}},
$$

where $M_{1}^{2}$ and $M_{2}^{2}$ are the Borel parameters in $\Sigma^{0}$ and $\Lambda$ channels. Since masses of the $\Sigma^{0}$ and $\Lambda$ are very close to each other, we will set $M_{1}^{2}=M_{2}^{2}=2 M^{2}$, hence $u_{0}=1 / 2$. It follows from Eq. (18) that in determining the $\Sigma^{0} \Lambda$ transition matrix moment one needs to know the residues $\lambda_{\Lambda}$ and $\lambda_{\Sigma^{0}}$. These residues are determined from baryon mass sum rules [4, 13].

$$
\begin{aligned}
M_{\Lambda} \lambda_{\Lambda}^{2} e^{-\frac{M_{\lambda}^{2}}{M^{2}}} & =\frac{m_{s}}{192 \pi^{4}}\left(-13+2 t+11 t^{2}\right) M^{6} E_{2}(x) \\
& +\frac{1}{48 \pi^{2}}(1-t)\{(13+11 t)\langle\bar{s} s\rangle+2(1+5 t)\langle\bar{q} q\rangle\} M^{4} E_{1}(x) \\
& -\frac{m_{0}^{2}}{16 \pi^{2}}\left(1-t^{2}\right)(2\langle\bar{s} s\rangle+\langle\bar{q} q\rangle) M^{2} E_{0}(x) \\
& +\frac{m_{s}}{18}\langle\bar{q} q\rangle\left\{\left(1+4 t-5 t^{2}\right)\langle\bar{s} s\rangle+3\left(5+2 t+5 t^{2}\right)\langle\bar{u} u\rangle\right\} \\
\lambda_{\Lambda}^{2} e^{-\frac{M_{\Lambda}^{2}}{M^{2}}}= & \frac{1}{256 \pi^{4}}\left(5+2 t+5 t^{2}\right) M^{6} E_{2}(x)
\end{aligned}
$$




$$
\begin{aligned}
& +\frac{1}{72}(1-t) m_{0}^{2}\langle\bar{q} q\rangle\{8(1+2 t)\langle\bar{s} s\rangle+(25+23 t)\langle\bar{q} q\rangle\} \frac{1}{M^{2}} \\
& +\frac{m_{s}}{96 \pi^{2}}\left\{3\left(5+2 t+5 t^{2}\right)\langle\bar{s} s\rangle+4\left(1+4 t-5 t^{2}\right)\langle\bar{q} q\rangle\right\} M^{2} E_{0}(x) \\
& +\frac{m_{s}}{16 \pi^{2}} m_{0}^{2}\langle\bar{q} q\rangle\left(1-t^{2}\right)\left\{\gamma_{E}-\ln \left(\frac{M^{2}}{\Lambda^{2}}\right)\right\} \\
& -\frac{1}{18}\langle\bar{q} q\rangle(1-t)\{2(1+5 t)\langle\bar{s} s\rangle+(13+11 t)\langle\bar{q} q\rangle\} \\
& -\frac{m_{s}}{96 \pi^{2}} m_{0}^{2}\left\{\left(5+2 t+5 t^{2}\right)\langle\bar{s} s\rangle+\left(-5+4 t+t^{2}\right)\langle\bar{q} q\rangle\right\}, \\
& M_{\Sigma^{0}} \lambda_{\Sigma^{0}}^{2} e^{-\frac{M_{\Lambda}^{2}}{M^{2}}}=\frac{m_{s}}{64 \pi^{2}}\left(1-t^{\prime}\right)^{2} M^{6} E_{2}(x) \\
& \left.+\frac{1}{16 \pi^{2}}\left(1-t^{\prime}\right)\left\{\left(-1+t^{\prime}\right)\langle\bar{s} s\rangle+6\left(1+t^{\prime}\right)\langle\bar{q} q\rangle\right)\right\} M^{4} E_{1}(x) \\
& \text { - } \frac{3}{16 \pi^{2}} m_{0}^{2}\langle\bar{q} q\rangle\left(1-t^{\prime 2}\right) M^{2} E_{0}(x) \\
& +\frac{m_{s}}{6}\langle\bar{q} q\rangle\left\{-3\left(-1+t^{2}\right)\langle\bar{s} s\rangle+\left(5+2 t^{\prime}+5 t^{\prime 2}\right)\langle\bar{q} q\rangle\right\}, \\
& \lambda_{\Sigma^{0}}^{2} e^{-\frac{M_{\Lambda}^{2}}{M^{2}}}=\frac{1}{256 \pi^{4}}\left(5+2 t^{\prime}+5 t^{\prime 2}\right) M^{6} E_{2}(x) \\
& +\frac{m_{s}}{32 \pi^{2}}\left\{\left(5+2 t^{\prime}+5 t^{2}\right)\langle\bar{s} s\rangle-12\left(-1+t^{\prime 2}\right)\langle\bar{q} q\rangle\right\} M^{2} E_{0}(x) \\
& +\frac{1}{24} m_{0}^{2}\langle\bar{q} q\rangle\left(1-t^{\prime}\right)\left\{12\left(1+t^{\prime}\right)\langle\bar{s} s\rangle+\left(-1+t^{\prime}\right)\langle\bar{q} q\rangle\right\} \frac{1}{M^{2}} \\
& +\frac{3 m_{s}}{16 \pi^{2}} m_{0}^{2}\langle\bar{q} q\rangle\left(1-t^{\prime 2}\right)\left\{\gamma_{E}-\ln \left(\frac{M^{2}}{\Lambda^{2}}\right)\right\} \\
& -\frac{m_{s}}{96 \pi^{2}} m_{0}^{2}\left\{\left(5+2 t^{\prime}+5 t^{\prime 2}\right)\langle\bar{s} s\rangle-3\left(-1+t^{\prime 2}\right)\langle\bar{q} q\rangle\right\} \\
& -\frac{1}{6}\langle\bar{q} q\rangle\left(1-t^{\prime}\right)\left\{6\left(1+t^{\prime}\right)\langle\bar{s} s\rangle+\left(-1+t^{\prime}\right)\langle\bar{q} q\rangle\right\},
\end{aligned}
$$

where $\Lambda$ is the QCD scale parameter and it is chosen to be $\Lambda=0.5 \mathrm{GeV}$.

In this set of expressions, Eqs. (19), (21) and (20), (22) correspond to the structures proportional to the unit operator and $\not p$, respectively. In order to obtain the transition magnetic moment $\mu_{\Sigma^{0} \Lambda}$, we substitute the values of $\lambda_{\Lambda}$ and $\lambda_{\Sigma^{0}}$ from Eqs. (20) and (22) into Eq. (18).

\section{Numerical analysis}

In this section we present the numerical analysis of the sum rules for the $\Sigma^{0} \Lambda$ transition matrix element which has already been obtained in the previous section. As can obviously be seen from Eq. (18) the main input parameters of the sum rules are photon wave functions. 
It was shown in $[15,16]$ that the leading photon wave functions receive only small corrections from the higher conformal spin, so that their deviation from asymptotic form is inessential. We shall use the following expressions for the photon wave functions $[15,17]$ :

$$
\begin{aligned}
\phi(u) & =6 u(1-u), \quad \psi(u)=1 \\
g_{1}(u) & =-\frac{1}{8}(1-u)(3-u), \quad g_{2}(u)=-\frac{1}{4}(1-u)^{2} .
\end{aligned}
$$

The values of input parameters that are used in the numerical calculations are: $f=$ $0.028 \mathrm{GeV}^{2}, \chi=-4.4 \mathrm{GeV}^{-2}$ [21] (in [22] it is estimated to be $\chi=-3.3 \mathrm{GeV}^{-2}$ ), $\langle\bar{q} q\rangle(1 \mathrm{GeV})=-(0.243)^{3} \mathrm{GeV}^{3}$, and $m_{0}^{2}=(0.8 \pm 0.2) \mathrm{GeV}^{2}[23], m_{s}(1 \mathrm{GeV})=(150 \pm$ 50) $\mathrm{MeV},\langle\bar{s} s\rangle(1 \mathrm{GeV})=0.8\langle\bar{q} q\rangle(1 \mathrm{GeV})$.

In further numerical analysis we set $t=t^{\prime}$. Since the transition magnetic moment is a physical quantity it must be independent of the parameters $t$, continuum threshold $s_{0}$ and Borel mass $M^{2}$. So the main problem is to find a region where the result of the transition magnetic moment is practically independent of the parameters $t, s_{0}$ and $M^{2}$.

In Fig. (1) we present the dependence of the transition magnetic moment on the Borel parameter $M^{2}$. The continuum threshold $s_{0}$ is determined from mass sum rules for $\Sigma$ and $\Lambda$ baryons [3, 14] and from $\Sigma^{0}-\Lambda$ mass difference sum rules [20]. It follows from this figure that the working region of Borel mass $M^{2}$ is $1 \mathrm{GeV}^{2} \leq M^{2} \leq 1.5 \mathrm{GeV}^{2}$. Moreover we see that for the choices $s_{0}=3 \mathrm{GeV}^{2}$ and $s_{0}=4 \mathrm{GeV}^{2}$, the variation in the results is about $10 \%$, i.e., the transition magnetic moment can be said to be practically insensitive to the value of the continuum threshold at $t=-3, t=-2$ and $t=3$. The result also seems to be practically independent of the choice of the value of the parameter $t$.

Before determining the transition magnetic moment, the next problem to be considered is to find an appropriate region of $t$. For this purpose we have used the mass sum rules (see Eqs. (19)-(21)). Two criteria should be met by the mass sum rules. First of all, each sum rule must separately be positive. After an analysis of the mass sum rules, we found that the region $-0.6 \leq t \leq 0.9$ is unphysical for $\Lambda$, and $-0.4 \leq t \leq 0.9$ is unphysical for $\Sigma^{0}$. The second criteria is that the predicted mass of the baryons, obtained by considering the ratio of the chiral odd and the chiral even mass sum rule, should be stable in regard to variations of the parameter $t$. In the early analysis of the mass sum rules, only the ratio is considered and it was found that the ratio stabilizes at $t=-0.2$, but as we have already noted, this value is not in the physical region. Our analysis shows that, the most appropriate value of $t$ is given by $-0.5 \leq \cos \theta \leq 0.5$ where $\theta$ is defined through the relation $t=\tan \theta$. This region of $\theta$ corresponds to $t \geq 1.7$ or $t \leq-1.7$. One should also note that the Ioffe current, which corresponds to the choice $t=-1$, is also not in the appropriate region.

In Fig. (2) the dependence of the transition magnetic moment on $\cos \theta$ at $M^{2}=1 \mathrm{GeV}^{2}$, $s_{0}=3 \mathrm{GeV}^{2}$ and $s_{0}=4 \mathrm{GeV}^{2}$ is depicted. A reasonable value for the transition magnetic moment must be obtained far from the unphysical region. We observe from Fig. (2) that $\mu_{\Sigma^{0} \Lambda}$ is quite stable in the region $-0.5 \leq \cos \theta \leq-0.5$ and practically seems to be independent of $\cos \theta$ (or $t$ ) and continuum threshold $s_{0}$. In this region of interest we obtain

$$
\left|\mu_{\Sigma^{0} \Lambda}\right|=(1.6 \pm 0.3) \mu_{N}
$$

for the transition magnetic moment, where the errors are mainly due to variations with respect to the Borel mass $M^{2}$, the continuum threshold $s_{0}$, the value of the parameters $\chi$, and omitted higher twist photon wave-function contributions. 
Finally let us compare our result on $\mu_{\Sigma^{0} \Lambda}$ with the results of the existing theoretical calculations and experimental data. For the transition magnetic moment $\mu_{\Sigma^{0} \Lambda}$ the traditional QCD sum rules predicts $\left|\mu_{\Sigma^{0} \Lambda}\right|=1.5 \mu_{N}$ [7]. The constituent quark model predicts that $\mu_{\Sigma^{0} \Lambda}=\left(\mu_{d}-\mu_{u}\right) / \sqrt{3}$, and with $\mu_{d}=-0.972 \mu_{N}$ and $\mu_{u}=1.852 \mu_{N}$, this result leads to $\mu_{\Sigma^{0} \Lambda} \simeq-1.65$. The experimental result of the transition magnetic moment is measured to be $\mu_{\Sigma^{0} \Lambda}=-1.6 \mu_{N}$ (see [24]). When we compare these results we see that our prediction on the transition magnetic moment is in a good agreement with those predicted by the traditional QCD sum rules and constituent quark model, as well as with the existing experimental data. 


\section{References}

[1] M. A. Shifman, V. I. Vainshtein, V. I. Zakharov, Nucl. Phys. B147 (1979) 385.

[2] L. J. Reinders, H. R. Rubinstein, S. Yazaki, Phys. Rev. C127 (1985) 1.

[3] B. L. Ioffe, Nucl. Phys. B188 (1981) 317; Nucl. Phys. B191 (1981) (E)591.

[4] V. Chung, H. G. Dosch, M. Kremer, D. Scholl, Nucl. Phys. B197 (1982) 55.

[5] B. L. Ioffe and A. V. Smilga, Nucl. Phys. B232 (1984) 109.

[6] I. I. Balitsky and A. V. Yung, Phys. Lett. B129 (1983) 328.

[7] S. L. Zhu, W. Y. P. Hwang and Z. S. Yang, Phys. Rev. D57 (1998) 1527.

[8] V. M. Braun, preprint: hep-ph/9801222 (1998).

[9] R. Rückl preprint: hep-ph/9810338 (1998);

Proc. "Varenna 1997 Heavy Flavor Physics" 265.

[10] V. M. Braun, I. E. Filyanov, Z. Phys. C44 (1989) 157.

[11] T. M. Aliev, A. Özpineci, M. Savci, Nucl. Phys. A678 (2000) 443.

[12] T. M. Aliev, A. Özpineci, M. Savci, Phys. Rev. D62 (2000) 053012.

[13] H. G. Dosch, M. Jamin, and S. Narison, Phys. Lett. B220 (1989) 251.

[14] I. I. Balitsky, V. M. Braun, Nucl. Phys. B311 (1988) 541.

[15] V. M. Braun, I. E. Filyanov, Z. Phys. C48 (1990) 239.

[16] I. I. Balitsky, V. M. Braun, A. V. Kolesnichenko, Nucl. Phys. B312 (1989) 509.

[17] A. Ali, V. M. Braun, Phys. Lett. B359 (1995) 223.

[18] V. M. Belyaev, V. M. Braun, A. Khodjamirian and R. Rückl, Phys. Rev. D51 (1995) 6177.

[19] T. M. Aliev, M. Savc1, Phys. Rev. D61 (2000) 0160008.

[20] W. Y. P. Hwang and K. C. Yang, Phys. Rev. D49 (1994) 460

[21] V. M. Belyaev, Ya. I. Kogan, Yad. Fiz. 40 (1984) 1035.

[22] I. I. Balitsky, A. V. Kolesnichenko, Yad. Fiz. 41 (1985) 282.

[23] V. M. Belyaev, B. L. Ioffe, JETP 56 (1982) 493.

[24] Particle Data Group, C. Caso et. al., Eur. Phys. Journal C15 (2000) 744. 


\section{Figure captions}

Fig. (1) The dependence of the transition magnetic moment $\left|\mu_{\Sigma^{0} \Lambda}\right|$ on the Borel mass $M^{2}$ at $t=-3 ; t=-2.0 ; t=+3$ and at the continuum threshold $s_{0}=3.0 \mathrm{GeV}^{2}$ and $s_{0}=4.0 \mathrm{GeV}^{2}$.

Fig. (2) The dependence of the transition magnetic moment $\left|\mu_{\Sigma^{0} \Lambda}\right|$ on $\cos \theta$ at $M^{2}=$ $1 \mathrm{GeV}^{2}, s_{0}=3 \mathrm{GeV}^{2}$ and $s_{0}=4 \mathrm{GeV}^{2}$. 


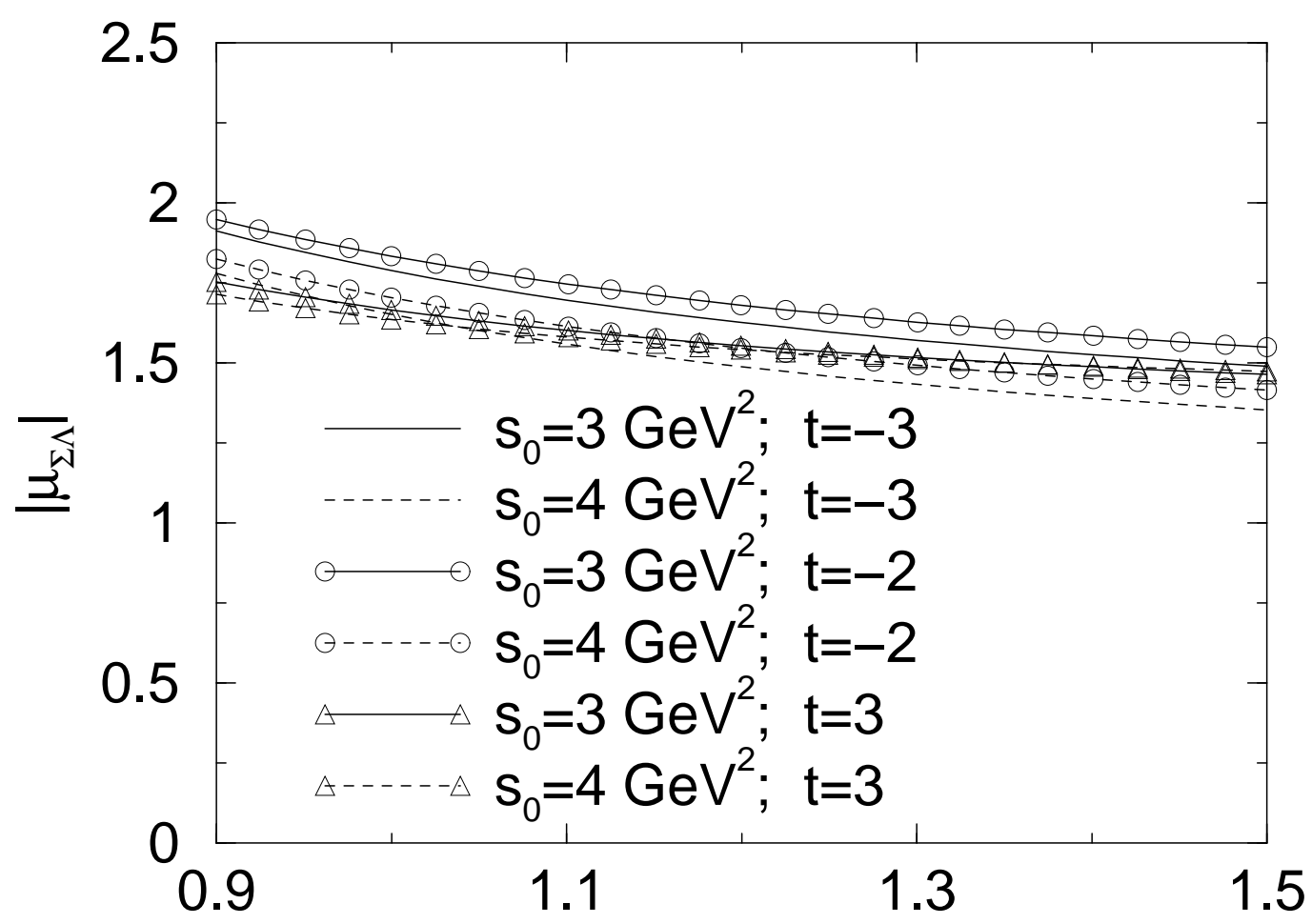

Figure 1

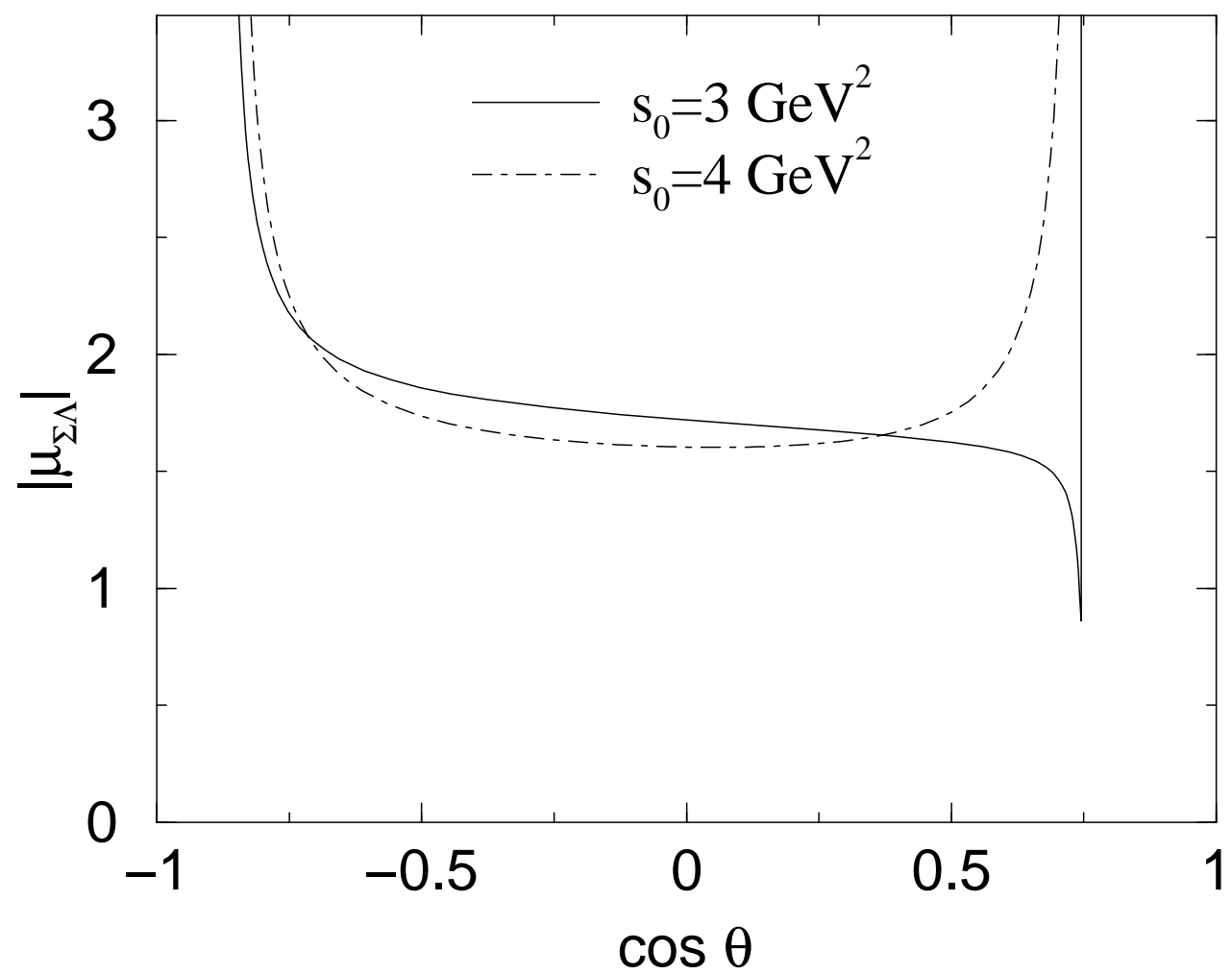

Figure 2 